\title{
O uso de plantas medicinais e o interesse pelo cultivo comunitário por moradores do bairro São Francisco, município de Pitangui, MG
}

\author{
The use of medicinal plants and the interest for community cultivation by \\ residents of the São Francisco neighborhood, Pitangui municipality, MG
}

El uso de plantas medicinales y el interés por el cultivo comunitario por habitantes del barrio São Francisco, municipio de Pitangui, MG

\author{
Filipe Rodrigues Valeriano ${ }^{1}$ \\ Fabiana Ramos Savani ${ }^{2}$ \\ Maria Rejane Valeriano da Silva ${ }^{3}$
}

Recebido em 16/02/2018; revisado e aprovado em 17/05/2018; aceito em 06/06/5018

DOI: http://dx.doi.org/10.20435/inter.v0i0.1846

\begin{abstract}
Resumo: O estudo foi realizado no bairro São Francisco, região urbana de Pitangui, Minas Gerais, com o propósito de investigar o conhecimento popular na utilização de plantas medicinais. As entrevistas semiestruturadas foram realizadas com 50 famílias residentes no bairro durante o período de julho a setembro de 2017. Foram citadas 53 espécies pertencentes a 29 famílias. As folhas foram a parte mais utilizada, e a principal forma de preparo é a infusão/chá.
\end{abstract}

Palavras-chave: Etnobotânica; quintais; recursos vegetais.

Abstract: The study has been carried out in the São Francisco neighborhood, urban area of Pitangui, Minas Gerais, with the objective of investigate popular knowledge in the use of medicinal plants. Semi-structured interviews were conducted with 50 families living in the neighborhood during the period between july to september 2017. 53 species belonging to 29 families were cited. The leaves were the most used part and the main form of preparation is the infusion/tea.

Keywords: Ethnobotany; home garden; plant resources.

Resumen: El estudio fue realizado en el barrio São Francisco, región urbana de Pitangui, Minas Gerais, con el propósito de investigar el conocimiento popular en la utilización de plantas medicinales. Las entrevistas semiestructuradas fueron realizadas con 50 familias residentes en el barrio durante el período julio a septiembre de 2017. Fueron citadas 53 especies pertenecientes a 29 familias. Las hojas fueron la parte más utilizada y la principal forma de preparación es la infusión / té.

Palabras clave: Etnobotánica; jardín; recursos vegetales.

\section{INTRODUÇÃO}

A utilização de plantas para fins medicinais não é algo recente, pelo contrário, possuí uma tradição milenar que vem sendo transferida entre as gerações ao longo do tempo. Essas práticas são observadas com maior frequência em populações tradicionais, como caiçaras e indígenas (MOREIRA et al., 2002), que utilizam esses recursos para finalidades curativas de seus males.

O uso por populações rurais é repassado, oralmente, entre gerações (MOREIRA et al., 2002). Consideradas como tradições, essas práticas são transmitidas para seus familiares, tendo um valor histórico, cultural e socioeconômico (MARTINS, 2016).

Para Brasileiro et al. (2008), na atualidade, muitas razões têm direcionado para o aumento do uso desses recursos, um deles é o preço elevado dos fármacos industrializados, a dificuldade

\footnotetext{
${ }^{1}$ Universidade Federal dos Vales do Jequitinhonha e Mucuri, Diamantina, Minas Gerais, Brasil.

${ }^{2}$ Universidade Federal do ABC, Santo André, São Paulo, Brasil.

${ }^{3}$ Faculdade de Pará de Minas, Pará de Minas, Minas Gerais, Brasil.
} 
das populações para obter auxílio médico e, segundo os autores, a propensão para o uso dos recursos naturais.

De acordo com Firmo et al. (2011), as utilidades das plantas são resultantes de um série de influências culturais, como a dos colonizadores europeus, indígenas e africanos. Porém, de maneira geral, o conhecimento popular é desenvolvido por grupamentos culturais que ainda convivem intimamente com a natureza, observando-a de perto no seu dia a dia e explorando suas potencialidades, mantendo vivo e crescente esse patrimônio pela experimentação sistemática e constante.

Hamilton (2003) ressalta que, "[...] as plantas medicinais representam a principal matéria médica utilizada pelas chamadas medicinas tradicionais, ou não ocidentais, em suas práticas terapêuticas, sendo a medicina popular a que utiliza o maior número de espécies diferentes".

Nesse contexto, em 2012, o Ministério da Saúde publicou o repasse do valor de R\$ 6,7 milhões para 12 municípios brasileiros, com o objetivo de fortalecer o programa nacional de plantas medicinais e fitoterápicos (BRASIL, 2012).

Dessa forma, o Brasil está buscando criar diretrizes na área de plantas medicinais utilizadas pela população urbana para fortalecer e oferecer assistência baseando-se no conhecimento popular e científico.

O presente estudo teve como objetivo investigar o conhecimento e a utilização de plantas medicinais por moradores do município de Pitangui, MG, no bairro de São Francisco. Especificamente o estudo se propôs a verificar a relação etnobotânica, bem como o interesse pelo cultivo de uma horta medicinal comunitária. Realizaram-se entrevistas semiestruturadas com 50 moradores, cada um representando uma família e, após o levantamento dos dados, procedeu-se à realização das análises quantitativa e qualitativamente. Os materiais relatados pelos entrevistados não foram coletados e herborizados, sendo identificados a partir da utilização de imagens para a confirmação do material e da realização da análise da nomenclatura através da literatura. Foi possível reconhecer o vasto conhecimento adquirido pelas populações através do conhecimento adquirido dos seus antecessores. Por outro lado, vale ressaltar que muitas das espécies mencionadas pelos entrevistados ainda não foram submetidas à validação, sendo necessária a correlação dos estudos científicos na área da farmacologia, a fim de obter informações com objetivo de gerar padronização quanto a formas de preparo, conservação do produto, entre outros, conforme será apresentado.

\section{MATERIAL E MÉTODOS}

O trabalho foi realizado no munícipio de Pitangui, MG, localizado ao centro-oeste do estado sob as coordenadas 190 40' 58" S e 44 53' 25" W e altitude de 709 m. Segundo o último censo do IBGE (IBGE, 2017) a população era de 25.311 habitantes em 2010, com uma estimativa 27.706 pessoas em 2017.

A cidade se encontra a $120 \mathrm{~km}$ da capital Belo Horizonte e apresenta um clima tropical de altitude. A classificação do clima é Aw de acordo com Köppen e Geiger (1928), apresentando uma temperatura média de $21.8^{\circ} \mathrm{C}$ e pluviosidade média anual de $1337 \mathrm{~mm}$.

Os dados do presente estudo foram coletados no período de julho a setembro de 2017, no bairro São Francisco, área urbana do Munícipio de Pitangui. A população estudada foi composta por 50 famílias $(n=50)$, sendo a entrevista realizada com o morador responsável da residência. 
As entrevistas foram realizadas a partir da utilização de questionários semiestruturados, compostos por 16 questões, tendo como base o trabalho de Ferreira e Godoy (2016), no intuito de mensurar questões socioeconômicas e questões relacionadas ao consumo de plantas medicinais.

Apenas um morador com no mínimo 18 anos foi entrevistado em cada domicílio (RODRIGUES; GUEDES, 2006; SANTOS; FARIA; VILHALVA, 2015). Ao final das entrevistas, sempre que permitido, foram feitos registros fotográficos dos quintais contendo algumas plantas cultivadas para posterior transcrição. Após o levantamento dos dados, procedeu-se à realização das análises quantitativa e qualitativamente. Os resultados foram exibidos por meio da média e citações de plantas por frequências relativas. As análises dos dados foram realizadas através do programa Microsoft Windows Excel $2007^{\circledR}$.

Os materiais relatados pelos entrevistados não foram coletados e herborizados, sendo identificados a partir da utilização de imagens para a confirmação do material e da realização da análise da nomenclatura através da literatura, como realizado por Almeida et al. (2009); Carvalho et al. (2011); Oliveira e Gondim (2013). Também foram utilizados como referência a lista de espécies registradas pela ANVISA (BRASIL, 2014) e o Manual de Plantas Medicinais (NASCIMENTO; VIEIRA, 2014).

\section{RESULTADOS E DISCUSSÃO}

A maioria das pessoas entrevistadas foi do sexo feminino, correspondendo em torno de $94 \%$ dos informantes questionados. Esse fato foi observado por outros autores (BORBA; MACEDO, 2006; MACEDO; OSHIIWA; GUARIDO, 2007; ALMEIDA et al., 2009; ALBERTASSE; THOMAZ; ANDRADE, 2010; GIRALDI; HANAZKI, 2010; COSTA; MAYWORM, 2011). Arnous, Santos e Beinner (2005) atribuíram à prevalência das mulheres ao fato de os homens estarem ausentes do domicílio no horário em que as entrevistas ocorreram. Silva e Faria (2014) ressaltam que as mulheres passam um período maior em suas residências devido a suas atividades domésticas, não se ausentando por muito tempo. Para Rodrigues e Casali (2002), os maiores detentores do conhecimento sobre as plantas medicinais são as mulheres que exercem um importante papel no processo de transmissão para as gerações vindouras. Oliveira, Kffuri e Casali (2010) em seu estudo ressaltam que, na maioria das famílias interrogadas, a mulher exercia um papel responsável no lar, sendo ela incumbida pelo cultivo e preparo de 'ervas medicinais'bem como por outras atividades relacionadas à alimentação e aos cuidados das crianças e familiares em ocasião de enfermidades.

Quanto à faixa etária, foi observado que a idade variou entre 19 a 84 anos, sendo a maioria dos entrevistados (38 pessoas) com idade acima dos 30 anos. Segundo Lima et al. (2000), as pessoas que mais se dispuseram a fornecer informações sobre a utilização de plantas medicinais, foram aquelas com faixa etária a partir dos 30 anos. Borba e Macedo (2006) ressaltam que as pessoas mais velhas são as principais fontes de informações.

A escolaridade declarada pela maioria dos respondentes foi de ensino fundamental incompleto (42\%), sendo constatado que a maioria não chegou a concluir nem a 4ạ série do ensino fundamental. Resultados análogos em algumas pesquisas etnobotânicas foram encontrados por: Moura e Andrade (2007); Carniello et al. (2010). Nobre (2015) infere que o nível de formação de uma população pode influir sobre as práticas comuns de uso e sobre o conhecimento das plantas medicinais, mesmo que esses métodos estejam intimamente ligados à tradição popular 
e aos conhecimentos empíricos.

As famílias do bairro são numerosas, apresentando entre um a dez moradores por habitação. Foi verificado que a maioria dos entrevistados apresentam 5 ou acima de 5 moradores por residência (Tabela 1).

Tabela 1 - Quantidade de moradores por residência (A) e renda familiar (B) dos moradores do bairro São Francisco, Pitangui, MG, 2017

\begin{tabular}{lclc}
\hline (A) Moradores por residência & $\%$ & (B) Renda Familiar & $\%$ \\
\hline 1 Pessoa & 10,0 & Menor 1 Salário & 4,0 \\
2 Pessoas & 12,0 & 1 Salário & 32,0 \\
3 Pessoas & 30,0 & 2 Salários & 34,0 \\
4 Pessoas & 14,0 & 3 Salários & 26,0 \\
5 ou acima de 5 Pessoas & 34,0 & Acima de 4 Salários & 4,0 \\
\hline
\end{tabular}

Fonte: Elaborada pelos autores com base em Ferreira e Godoy (2016).

A renda familiar é observada na Tabela 1, sendo possível verificar que a maioria dos moradores (34\%) apresentou renda igual a dois salários mínimos. Segundo IBGE (2017), em 2017, a média salarial por mês era 1,7 salário mínimo no município.

Em relação aos conhecimentos e usos das plantas medicinais, apenas quatro pessoas (8\%) afirmaram não usar plantas medicinais. O costume de utilizar essas fontes para o preparo de 'remédios' foi evidenciado por 92\% dos entrevistados. Esse resultado revela que o uso de plantas medicinais é uma prática ainda comum entre a população atual, fato esse também observado por Lucena et al. (2013); Bernardes (2012).

Outra informação relevante é que a maioria das pessoas (44\%) possui o hábito de cultivar as plantas medicinais nos quintais de suas residências (Figura 1).

Figura 1 - Formas de obtenção de plantas medicinais por moradores do bairro São Francisco, Pitangui, MG, 2017

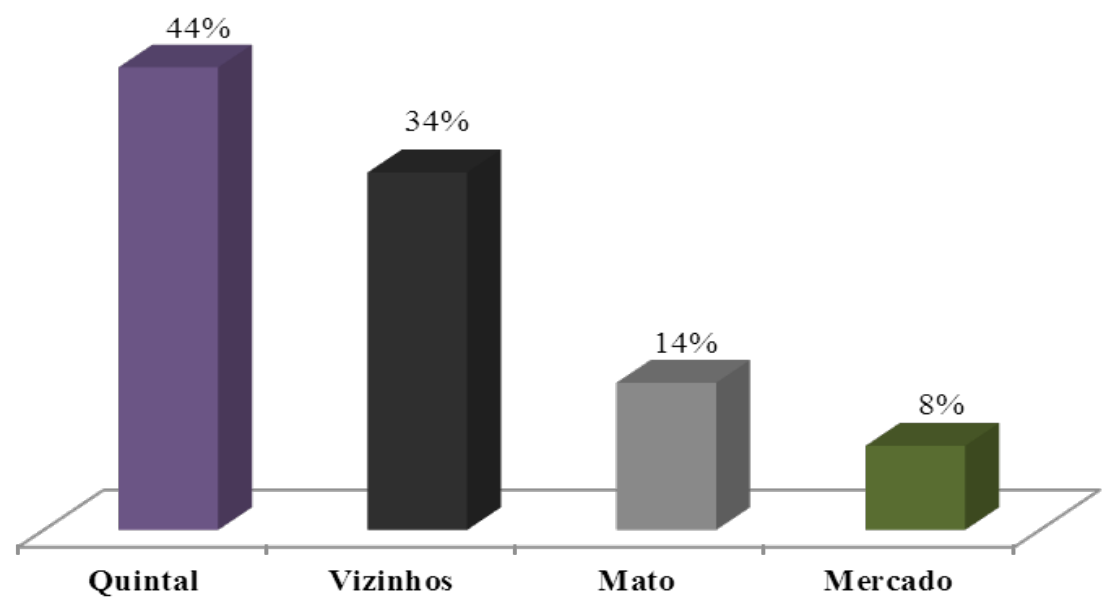

Fonte: Elaborada pelos autores com base em Ferreira e Godoy (2016).

Os quintais urbanos têm sido citados como importantes sistemas agroflorestais, apresentando grande diversidade de espécies medicinais utilizadas para tratamentos (SIVIERO et al., 2012; 
FREITAS et al., 2012). Além disso, contêm plantas ornamentais e alimentícias, tornando-se uma opção de incremento na renda familiar, uma vez que elas poderão ser colhidas e comercializadas entre os moradores do bairro ou em feira livre que ocorre todos os finais de semana no município (MEIRELLES et al., 2003; FREITAS et al., 2010).

Quando indagados sobre quem ensinou a utilizarem plantas medicinais, grande parte dos informantes, afirmou ter aprendido com seus ascendentes familiares (84\%), sendo as mães (52\%) e avós (30\%) as principais responsáveis (Figura 2).

Figura 2-Origem do conhecimento sobre o uso de plantas medicinais por moradores do bairro São Francisco, Pitangui, MG, 2017

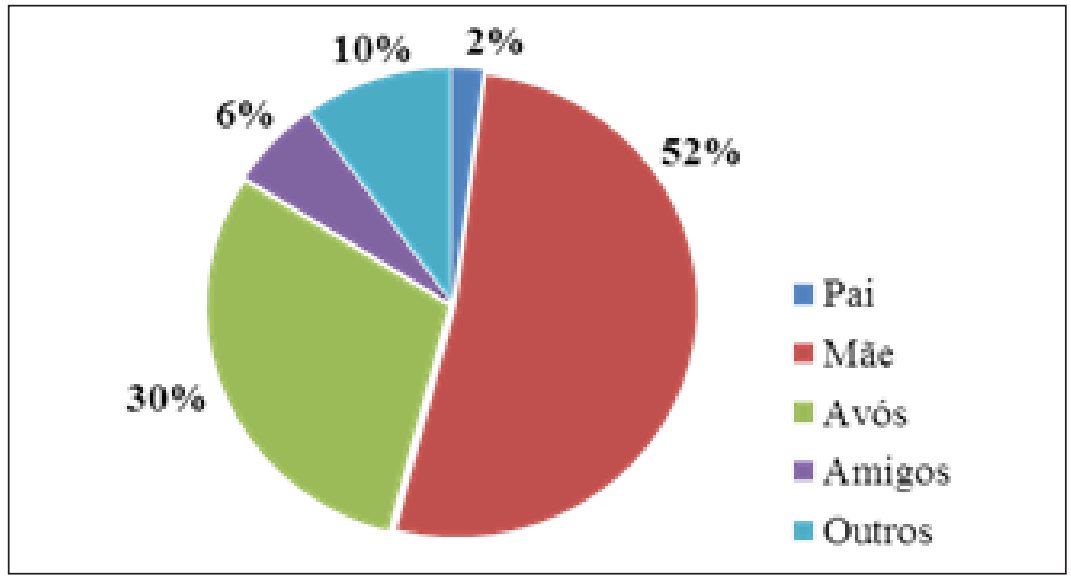

Fonte: Elaborada pelos autores com base em Ferreira e Godoy (2016).

Lucena et al. (2013) também observaram resultados parecidos, concluindo que a principal fonte de transmissão do conhecimento era através dos ascendentes familiares.

Quanto à frequência de utilização das plantas para fins medicinais, foi observada que boa parte dos moradores do bairro São Francisco (68\%), faz uso das plantas medicinais somente em situações de desconforto (Figura 3).

Figura 3 - Frequência de utilização de plantas medicinais por moradores do bairro São Francisco, Pitangui, MG, 2017

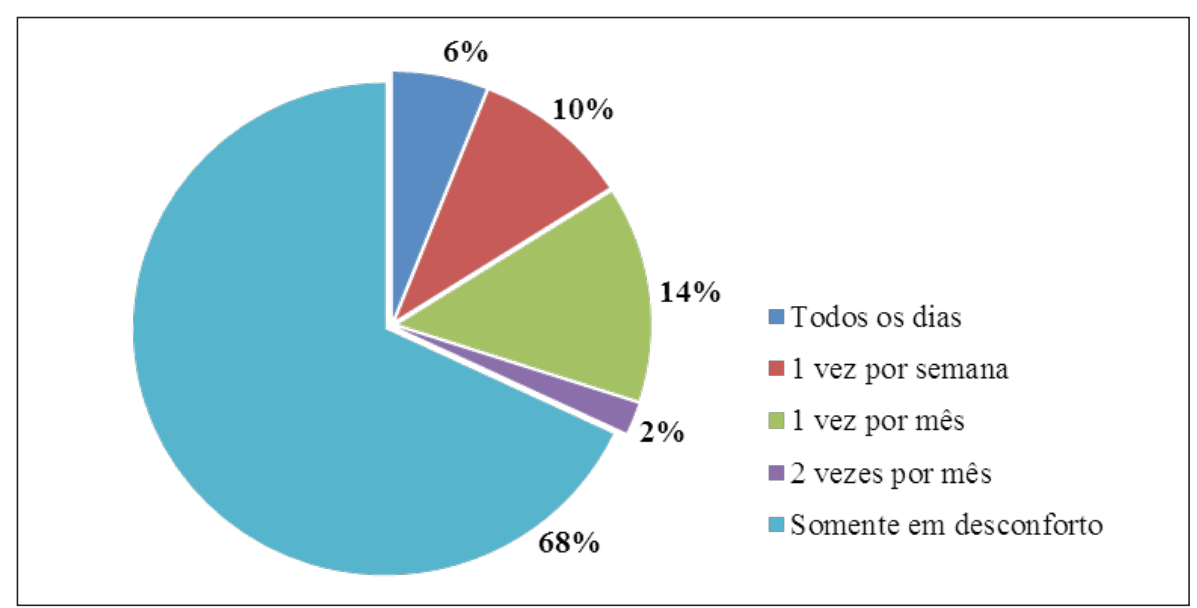

Fonte: Elaborada pelos autores com base em Ferreira e Godoy (2016). 
Também foi constatado o uso sistemático, sendo utilizadas todos os dias (6\%), uma vez por semana (10\%), uma vez ao mês (14\%) e duas vezes por mês (2\%). Essa ultima fração tem relacionado o uso das plantas medicinais também a questões de 'gosto pessoal', sendo usado como um substituto do café.

Quando questionados sobre o efeito das plantas medicinais, 76\% consideram o efeito dessas plantas confiáveis, e 18\%, muito confiável, enquanto $4 \%$ responderam indiferentes e apenas $2 \%$ não consideraram o uso confiável (Tabela 2A).

Tabela 2 - Confiança na ação das plantas medicinais (A) e maior eficácia delas em relação aos medicamentos (B), por moradores do bairro São Francisco, Pitangui, MG, 2017

\begin{tabular}{lclc}
\hline (A) Confiança nas Plantas Medicinais & \% & (B) Eficácia das Plantas Medicinais & $\%$ \\
\hline Não Confiável & 2,0 & Discorda & 6,0 \\
Pouco Confiável & 0,0 & Discorda Parcialmente & 4,0 \\
Indiferente & 4,0 & Indeciso & 8,0 \\
Confiável & 76,0 & Concorda Parcialmente & 54,0 \\
Muito Confiável & 18,0 & Concorda & 28,0 \\
\hline
\end{tabular}

Fonte: Elaborada pelos autores com base em Ferreira e Godoy, 2016.

A partir dos relatos, foi verificada a permanência da crença popular, em que a maioria das pessoas associa o uso de produtos naturais à não ocorrência de efeitos colaterais. Associações semelhantes a essas foram encontradas por (OLIVEIRA; MENINI NETO, 2012; FERREIRA; GODOY, 2016).

Outro questionamento importante foi referente às plantas medicinais serem mais eficazes que os remédios farmacêuticos (Tabela 2B), com que 54\% concordaram parcialmente, alegando que as plantas medicinais são eficazes em algumas situações e em outras não. Ainda afirmaram que as respostas obtidas a partir das plantas são mais demoradas, sendo necessário, uso conciliado com medicamentos químicos.

Dentre os entrevistados, 74\% relataram que 'às vezes' aconselham os jovens a utilizarem plantas medicinais (Tabela 3A), não sendo ainda um hábito frequente (14\%).

Tabela 3 - Frequência com que os entrevistados aconselham as pessoas mais jovens a utilizarem plantas medicinais (A) e as partes mais utilizadas das plantas (B), por moradores do bairro São Francisco, Pitangui, MG, 2017

\begin{tabular}{lclc}
\hline (A) Aconselha Pessoas mais Jovens & $\mathbf{\%}$ & (B) Parte mais Utilizada das Plantas & $\%$ \\
\hline Nunca & 6,0 & Raiz & 8,0 \\
Raramente & 4,0 & Caule ou Casca & 4,0 \\
Às vezes & 74,0 & Folha & 80,0 \\
Frequentemente & 14,0 & Fruto & 6,0 \\
Muito Frequente & 2,0 & Semente ou Flores & 2,0 \\
\hline
\end{tabular}

Fonte: Elaborada pelos autores com base em Ferreira e Godoy, 2016.

Esses resultados foram diferentes quando comparados com os de Ferreira e Godoy (2016), que observou esse aconselhamento ocorrendo com uma maior frequência (40\%), atestando a essencialidade da propagação desse saber empírico e a transmissão do conhecimento entre 
gerações (SILVA et al., 2015). Demonstrando que para a manutenção da sabedoria popular sobre o meio que o cerca, faz-se necessário o envolvimento e o interesse das novas gerações (NOBRE, 2015).

As partes da planta mais utilizadas para o preparo dos medicamentos foram as folhas (80\%), seguida pela raiz (8\%) (Tabela 3B). Resultados similares foram encontrados por Lima (2000), Fuck et al. (2005), Borba e Macedo (2006), Lima et al. (2000), Almeida et al. (2009), Giraldi e Hanazaki (2010), Oliveira, Kffuri e Casali (2010). Segundo Castellucci et al. (2000), as folhas das plantas medicinais são mais utilizadas que todas as outras partes da planta, devido à facilidade para realizar a coleta. Além disso, os autores salientam que nas folhas se concentram a maior parte dos princípios ativos. Ademais, é importante ressaltar que a maioria das plantas utilizadas para tratamentos de moléstias são de hábitos herbáceos, apresentando folhas durante todo o ano (FREITAS et al., 2012) sendo, portanto, fácil adquiri-las.

Sobre o preparo, a forma predominante neste estudo foi a infusão/chá, representando $82 \%$ dos entrevistados. Esses resultados foram similares em outros estudos etnobotânicos como os encontrados por Arnous, Santos e Beinner (2005), Fuck et al. (2005), Cunha e Bortolotto (2011), Silva et al. (2012) Battisti et al. (2013). Ao serem questionados sobre a dosagem utilizada, a maioria afirmou não existir, sendo preparadas quantidades que possam ser tomadas de forma fracionada durante o dia.

Foram relatadas 53 plantas de uso medicinal conhecido pela população entrevistada, que estão distribuídas em 29 famílias botânicas (Tabela 4).

Tabela 4 - Plantas usadas como remédio por moradores do bairro São Francisco, Pitangui, MG, 2017, (P.U: parte utilizada)

\begin{tabular}{l|l|l|l|l|l}
\hline \multicolumn{1}{c}{ Nome científico } & Nome popular & \multicolumn{1}{c}{ Família } & \multicolumn{2}{c}{ Uso popular } \\
\hline $\begin{array}{l}\text { Achyrocline satureioides } \\
\text { (Lam.) DC. }\end{array}$ & Marcela & Asteraceae & $\begin{array}{l}\text { Dores no } \\
\text { estomago, barriga, }\end{array}$ & Antioxidante & $\begin{array}{l}\text { Folhas, } \\
\text { caule }\end{array}$ \\
\hline $\begin{array}{l}\text { Allium cepa var. aggregatum } \\
\text { G.Don }\end{array}$ & Cebola & Amaryllidaceae & Gripe & Sistema respiratório & Caule \\
\hline Allium sativum L. & Alho & Liliacea & Gripe & Sistema circulatório & Caule \\
\hline $\begin{array}{l}\text { Aloe vera (L.) Burm.f. } \\
\text { Arnica montana L. }\end{array}$ & Babosa & Asphodelaceae & $\begin{array}{l}\text { Melhora a pele e } \\
\text { o cabelo }\end{array}$ & Sistema circulatório & Folhas \\
\hline $\begin{array}{l}\text { Arrabidaea brachypoda } \\
\text { Bureau }\end{array}$ & $\begin{array}{l}\text { Cervejinha do } \\
\text { Mato }\end{array}$ & Aquifoliaceae & Problemas de rins & $\begin{array}{l}\text { Ação diurética, } \\
\text { depurativa }\end{array}$ & Folhas \\
\hline Arundo donax L. & Losna & Asteraceae & Dor no estomago & $\begin{array}{l}\text { Sistema } \\
\text { imunológico }\end{array}$ & Folhas \\
\hline Carica papaya L. & $\begin{array}{l}\text { Caninha do } \\
\text { reino }\end{array}$ & Poaceae & Gripe e tosse & Sistema respiratório & Folhas \\
\hline Cissus sicyoides L. & Mamão & Caricaceae & $\begin{array}{l}\text { Dor no estomago, } \\
\text { gripe }\end{array}$ & $\begin{array}{l}\text { Regula intestino, } \\
\text { sistema digestório }\end{array}$ & $\begin{array}{l}\text { Folhas, } \\
\text { flor }\end{array}$ \\
\hline Citrus acida & $\begin{array}{l}\text { Insulina do } \\
\text { mato }\end{array}$ & Vitaceae & Diabete & Sistema endócrino & Folhas \\
\hline
\end{tabular}




\begin{tabular}{|c|c|c|c|c|c|}
\hline Nome científico & Nome popular & Família & Uso popular & Literatura & P.U \\
\hline Coix lacryma-jobi L. & \begin{tabular}{|l|} 
Conta de \\
lágrima
\end{tabular} & Poaceae & Problemas de rins & Reumatismo & Folhas \\
\hline Cotyledon orbiculate L. & Balsamo & Crassulaceae & $\begin{array}{l}\text { Queimação no } \\
\text { estomago }\end{array}$ & Sistema circulatório & Folhas \\
\hline $\begin{array}{l}\text { Cymbopogon citratus } \\
\text { Andropogon cerifer Hack. }\end{array}$ & $\begin{array}{l}\text { Erva de } \\
\text { Príncipe }\end{array}$ & Poaceae & Gripe & Sistema respiratório & Folhas \\
\hline $\begin{array}{l}\text { Dasyphyllum tomentosum } \\
\text { (Spreng.) Cabrera }\end{array}$ & $\begin{array}{l}\text { Cambará de } \\
\text { Espinho }\end{array}$ & Verbenaceae & Gripe & Sistema respiratório & Folhas \\
\hline Dorstenia brasiliensis Lam. & Carapiá & Moraceae & Bronquite & Sistema digestório & Folhas \\
\hline $\begin{array}{l}\text { Ambrina ambrosioides (L.) } \\
\text { Spach }\end{array}$ & $\begin{array}{l}\text { Erva Santa } \\
\text { Maria }\end{array}$ & Chenopodiaceae & $\begin{array}{l}\text { Dores musculares, } \\
\text { verme }\end{array}$ & Sistema circulatório & Folhas \\
\hline $\begin{array}{l}\text { Echinodorus grandiflorus } \\
\text { (Cham. \& Schltdl.) Micheli }\end{array}$ & $\begin{array}{l}\text { Chapéu de } \\
\text { Couro }\end{array}$ & Alismataceae & \begin{tabular}{l|} 
Limpar o sangue, \\
problemas de rins \\
\end{tabular} & Sistema circulatório & Folhas \\
\hline Foeniculum vulgare Mill. & Funcho & Apiaceae & $\begin{array}{l}\text { Dores no } \\
\text { estomago, barriga, } \\
\text { cólica, calmante, } \\
\text { bronquite }\end{array}$ & $\begin{array}{l}\text { Sistema } \\
\text { imunológico }\end{array}$ & Folhas \\
\hline Gossypium hirsutum L. & Algodão & Malvaceae & Anti-inflamatório & Sistema endócrino & Folhas \\
\hline Ilex paraguariensis A.St.-Hil. & Mate & Aquifoliaceae & - & Sistema nervoso & Folhas \\
\hline Impatiens episcopi H.J.Veitch & Beijo Branco & Balsaminaceae & Anti-inflamatório & Sistema circulatório & Flor \\
\hline Kalanchoe pinnata (Lam.) Pers. & Folha Santa & Celastraceae & Dores nos ossos & Anti-inflamatório & Folhas \\
\hline Laurus nobilis L. & Louro & Lauraceae & Gripe & Sistema circulatório & Folhas \\
\hline Leonurus sibiricus L. & Macaé & Lamiaceae & Dor de cabeça & $\begin{array}{l}\text { Sistema digestório } \\
\text { e circulatório }\end{array}$ & Folhas \\
\hline Matricaria chamomilla L. & Camomila & Asteraceae & Calmante & Sistema nervoso & Flor \\
\hline Melissa officinalis L. & Erva-Cidreira & Lamiaceae & Calmante, insônia & $\begin{array}{l}\text { Diurética, } \\
\text { expectorante, } \\
\text { insônia nervosa }\end{array}$ & Folhas \\
\hline $\begin{array}{l}\text { Mentha abruptifolia Borbás ex } \\
\text { Heinr.Braun }\end{array}$ & Hortelã & Lamiaceae & $\begin{array}{l}\text { Expectorante, } \\
\text { dor de barriga, } \\
\text { gastrite, verme }\end{array}$ & Expectorante & Folhas \\
\hline Mentha pulegium L. & Poejo & Lamiaceae & Gripe, cólica & $\begin{array}{l}\text { Sistema } \\
\text { imunológico }\end{array}$ & Folhas \\
\hline Mentha viridis (L.) L. & Alevante & Lamiaceae & Gripe & $\begin{array}{l}\text { Sistema } \\
\text { imunológico }\end{array}$ & Folhas \\
\hline Mikania glomerata Spreng & Guaco & Asteraceae & Gripe & Sistema respiratório & Folhas \\
\hline Morus alba $\mathrm{L}$. & Amora & Moraceae & $\begin{array}{l}\text { Sintomas da } \\
\text { menopausa }\end{array}$ & Sistema endócrino & Folhas \\
\hline Nasturtium officinale R. Br. & Agrião & Brassicaceae & Gripe & $\begin{array}{l}\text { Sistema } \\
\text { imunológico }\end{array}$ & Folhas \\
\hline $\begin{array}{l}\text { Billbergia variegata (Arruda) } \\
\text { Schult. \& Schult.f. }\end{array}$ & Gravatá & Bromeliáceae & Bronquite & Sistema respiratório & Folhas \\
\hline Ocimum basilicum $\mathrm{L}$. & Manjericão & Lamiaceae & Gripe & Sistema nervoso & Folhas \\
\hline Passiflora zamorana Killip & Maracujá & Passifloraceae & Calmante & Sistema nervoso & Folhas \\
\hline Persea americana Mill. & Abacate & Lauraceae & Tosse e roquidão & Expectorante & Folhas \\
\hline Petroselinum crispum & Salsa & Apiaceae & $\begin{array}{l}\text { Gripe, infecção, } \\
\text { anti-inflamatório, } \\
\text { limpar o útero }\end{array}$ & $\begin{array}{l}\text { Sistema respiratório } \\
\text { e imunológico }\end{array}$ & Folhas \\
\hline $\begin{array}{l}\text { Petroselinum crispum (Mill.) } \\
\text { Fuss }\end{array}$ & Boldo & Lamiaceae & $\begin{array}{l}\text { Dores no } \\
\text { estomago, barriga, } \\
\text { cabeça, gripe }\end{array}$ & $\begin{array}{l}\text { Afecções do fígado } \\
\text { e do estômago, } \\
\text { cistite, constipação }\end{array}$ & Folhas \\
\hline Phyllanthus niruri & Quebra-pedra & Phyllanthaceae & Problemas de rins & Diurético & Folhas \\
\hline
\end{tabular}




\begin{tabular}{|c|c|c|c|c|c|}
\hline Nome científico & Nome popular & Família & Uso popular & Literatura & P.U \\
\hline Pimpinella anisum & Erva-doce & Apiaceae & $\begin{array}{l}\text { Calmante, dor de } \\
\text { barriga }\end{array}$ & Sistema digestório & Folhas \\
\hline Plantago major L. & Tansagem & Plantaginaceae & $\begin{array}{l}\text { Infecção, anti- } \\
\text { inflamatório }\end{array}$ & Anti-hemorrágico & $\begin{array}{l}\text { Folhas, } \\
\text { caule e } \\
\text { raiz }\end{array}$ \\
\hline $\begin{array}{l}\text { Polygala cyparissias A. St.-Hil. } \\
\& \text { Moq. }\end{array}$ & Vick & Polygalaceae & Bronquite, tosse & Sistema respiratório & Folhas \\
\hline Polygala paniculata $\mathrm{L}$. & $\begin{array}{l}\text { Vassourinha } \\
\text { de São Pedro }\end{array}$ & Polygalaceae & Diarreia e náuseas & Sistema respiratório & Folhas \\
\hline Psidium guajava $\mathrm{L}$. & Goiaba & Myrtaceae & Calmante, diarreia & Analgésico & Folhas \\
\hline Punica nana $\mathrm{L}$. & Romã & Lythraceae & $\begin{array}{l}\text { Gripe, anti- } \\
\text { inflamatório }\end{array}$ & $\begin{array}{l}\text { Sistema } \\
\text { imunológico }\end{array}$ & Fruto \\
\hline Rosa chinensis Jacq. & Rosa pequena & Rosaceae & Limpar o útero & Sistema nervoso & Flor \\
\hline Rosmarinus officinalis L. & Alecrim & Lamiaceae & $\begin{array}{l}\text { Problemas de } \\
\text { pressão, coração, } \\
\text { calmante, gripe }\end{array}$ & $\begin{array}{l}\text { Sistema } \\
\text { imunológico }\end{array}$ & Folhas \\
\hline Salvia officinalis L. & Salvia & Lamiaceae & $\begin{array}{l}\text { Problemas de } \\
\text { coração }\end{array}$ & Sistema endócrino & Folhas \\
\hline $\begin{array}{l}\text { Stryphnodendron adstringens } \\
\text { (Mart.) Coville }\end{array}$ & Barbatimão & Fabaceae & $\begin{array}{l}\text { Cicatrizante, dor } \\
\text { de cabeça }\end{array}$ & Sistema circulatório & Caule \\
\hline Zingiber officinale Roscoe & Gengibre & Zingiberaceae & Infecção & $\begin{array}{l}\text { Controle do mau } \\
\text { colesterol }\end{array}$ & Raiz \\
\hline
\end{tabular}

Fonte: Elaborada pelos autores com base em Ferreira e Godoy (2016).

As famílias com maior riqueza de espécies foram Lamiaceae (34,4\%), Asteraceae (17,2\%), Poaceae (10,3\%), Apiaceae (10,3\%) e o restante correspondente a 27,8\%. A predominância dessas famílias também foi observada em alguns trabalhos realizados por Castelucci et al. (2000), Dorigoni et al. (2001), Di Stasi et al. (2002), Hanazaki et al (2000), Pilla, Amorozo e Furlan (2006), Ferreira e Godoy (2016). Esses resultados confirmam a relevância que as duas famílias, Lamiaceae e Asteraceae, apresentam para as comunidades (PILLA; AMOROZO; FULAN, 2006), sendo conhecidas em todo o mundo como provedoras de plantas medicinais (DI STASI et al., 2002).

As 10 plantas com maior frequência (Figura 9) de utilização foram o boldo (Peumus boldus), seguido pela erva-cidreira (Melissa officinalis), hortelã (Mentha piperita), marcela (Achyrocline satureoides), alecrim (Rosmarinus officinalis), tanchagem (Plantago major), poejo (Mentha pulegium), erva-doce (Foeniculum vulgare), gengibre (Zingiber officinale), folha de goiaba (Psidium guajava). Veiga Junior (2008) e Pilla, Amorozo e Furlan (2006) apresentaram resultados em que foi verificado que o boldo foi uma das plantas mais frequentemente utilizadas. Essa espécie tem sido utilizada principalmente para tratamentos de afecções do fígado e do estômago, apresentando seus efeitos comprovados (PILLA; AMOROZO; FURLAN, 2006) e agindo principalmente na diminuição da acidez e do volume da secreção do suco gástrico (FISCHMAN et al., 1991) estando parte em concordância quanto às recomendações do uso habituado pela população.

Já para a erva-cidreira, seus principais usos pelos informantes locais foram para os tratamentos contra a ansiedade, sendo muito utilizada como calmante. Nascimento e Vieira (2014) reportaram os efeitos da erva-cidreira, sendo esta indicada como um sedativo leve e para tratamentos de insônia (MACEDO; OSHIIWA; GUARIDO, 2007).

A maioria das espécies utilizadas no bairro São Francisco, apresentava o hábito herbáceo (67\%), seguido pelo hábito arbustivo (20\%) e por ultimo arbóreo (13\%). 
Os maiores números de citações da utilização de plantas medicinais como recursos terapêuticos para o tratamento de moléstias foram para os casos de Gripe/Resfriado (21,4\%), seguido para tratamentos de ansiedade $(9,5 \%)$ e dores estomacais $(9,5 \%)$ (Figura 4$)$.

Figura 4 - Principais moléstias tratadas com o uso de plantas medicinais por moradores do bairro São Francisco, Pitangui, MG, 2017.

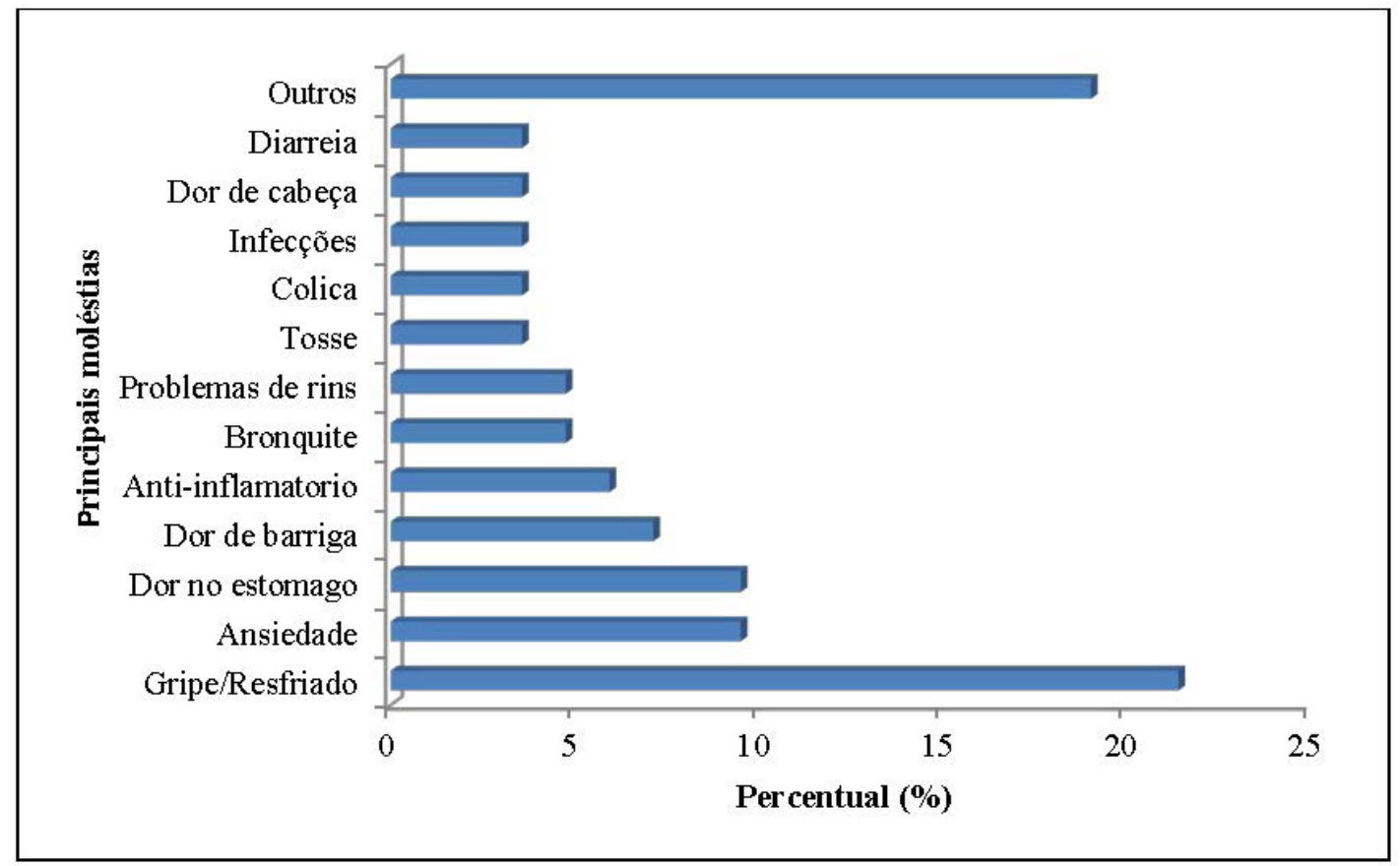

Fonte: Elaborada pelos autores com base em Ferreira e Godoy (2016).

Em alguns estudos de uso popular de plantas medicinais, alguns autores como Santos et al. (2016) também constataram que a maioria das espécies foram indicadas para o combate à gripe. Freitas et al. (2015) afirmam que um dos principais agravantes de saúde citados foi a gripe. 0 referido autor associa tais casos de gripe à possibilidade de esses agravos ocorrerem com maior regularidade, e devido à crença de que essas moléstias não precisam de atendimento médico.

Verificou-se que 92\% da população entrevistada demostrou interesse pelo cultivo de horta comunitária com plantas medicinais. Arnous, Santos e Beinner (2005) observaram o mesmo quando investigaram o interesse por cultivo comunitário no munícipio de Datas, MG. O referido autor preconiza a necessidade de profissionais da área da saúde serem capacitados por instituições provedoras do conhecimento em fitoterápicos e no uso de plantas medicinais, a fim de auxiliar a comunidade no desenvolvimento de boas práticas para o cultivo e conservação das espécies, resgates dos valores tradicionais e melhoria da saúde comunitária a partir dos produtos de baixo custo. Para isso, é preciso não apenas a integração da comunidade motivada, mas também a ação do Sistema Público, em conjunto com arcabouço legislativo de Plantas Medicinais e Fitoterápicos (Decreto n. 5.813, de 22 de junho de 2006) e os gestores públicos (prefeitura), no intuito de fornecer suporte para o desenvolvimento do cultivo.

Em 2010, a partir da Portaria n. 886, o Ministério da Saúde instituiu a 'Farmácia Viva' ao Sistema Único de Saúde (SUS), um programa de assistência social farmacêutico cujo intuito, além de incentivar o uso, é fornecer não apenas o estabelecimento de metodologias para o cultivo, mas todo o processamento, manipulação, armazenamento e a dispensação de preparações de 
plantas medicinais para a população (BRASIL, 2010; BRASIL, 2013). Projetos desse âmbito vêm acontecendo em alguns municípios de Minas Gerais, como a 'Farmácia Viva', no município de Betim, e 'Farmácia Verde', em Belo Horizonte, e têm promovido resultados positivos através da melhoria de vida e educação ambiental.

\section{CONCLUSÕES}

A realização deste estudo permitiu constatar alguns aspectos importantes pelos moradores do bairro, como o conhecimento e uso de diversas plantas medicinais e suas formas de obtenção, sugerindo uma riqueza de saber que vem sendo transmitida através da oralidade de geração a geração. Em contrapartida, muitas das espécies citadas pelos moradores ainda não foram submetidas à validação, e a utilização desses recursos não deve ser feita apenas de maneira não comprovada. Assim, é necessária a correlação dos estudos científicos na área da farmacologia, a fim de obter informações com objetivo de gerar padronização quanto formas de preparo, conservação do produto, entre outros aspectos relevantes, com a intenção de reduzir os riscos ocasionados pelo uso de forma errônea das dosagens, o que pode se tornar prejudicial à saúde.

\section{REFERÊNCIAS}

ALBERTASSE, P. D; THOMAZ, L. D; ANDRADE, M. A. Plantas medicinais e seus usos na comunidade da Barra do Jucu, Vila Velha, ES. Revista Brasileira de Plantas Medicinais, Paulínia, v. 12, n. 3, p. 250-60, 2010.

ALMEIDA, N. F. L; SILVA, S. R. S; SOUZA, J. M ; QUEIROZ, A. P. N; MIRANDA, G. S; OLIVEIRA, H. B. Levantamento etnobotânico de plantas medicinais na cidade de Viçosa-MG. Revista Brasileira de Farmacognosia, Curitiba, v. 90, n. 4, p. 316-20, 2009.

ARNOUS, A. H; SANTOS, A. S.; BEINNER, R. P. C. Plantas medicinais de uso caseiro-conhecimento popular e interesse por cultivo comunitário. Revista Espaço para a Saúde, Londrina, v. 6, n. 2, p. 1-6, 2005.

BATTISTI, C.; GARLET, T. M. B.; ESSI, L.; HORBACH, R. K.; ANDRADE, A.; BADKE, M. R. Plantas medicinai utilizadas no município de Palmeiras das Missões, RS, Brasil. Revista Brasileira de Biociências, Porto Alegre, v. 11, n. 3, p. 338-48, 2013.

BERNARDES, A. O. Pesquisa na escola: utilização de plantas medicinais pela comunidade escolar. Educação Pública, Rio de Janeiro. mar. 2012. Disponível em: http://www.educacaopublica.rj.gov.br/suavoz/0149. html. Acesso em: 22 jun. 2017.

BORBA, A. M.; MACEDO, M. Plantas medicinais usadas para a saúde bucal pela comunidade do bairro Santa Cruz, Chapada dos Guimarães, MT, Brasil. Acta Botânica Brasilica, Belo Horizonte, v. 20, n. 4, p. 771-82, 2006.

BRASIL. Agência Nacional de Vigilância Sanitária (ANVISA). RDC n. 26, de 13 de maio de 2014. Dispõe sobre o registro de medicamentos fitoterápicos e o registro e a notificação de produtos tradicionais fitoterápicos. Diário Oficial da União, Brasília, DF, 14 maio 2014, seção 1, p. 52.

BRASIL. Agência Nacional de Vigilância Sanitária (ANVISA). RDC n. 18, de 3 de abr. 2013. Dispõe sobre as boas práticas de processamento e armazenamento de plantas medicinais em farmácias vivas no âmbito do Sistema Único de Saúde (SUS). Diário Oficial da União, Brasília, DF, 5 abr. 2013, seção 1, p. 67.

BRASIL. Ministério da Saúde. Portaria n. 886, de 20 abril de 2010. Institui a Farmácia Viva no âmbito do Sistema Único de Saúde (SUS). Diário Oficial da União, Brasília, DF, 22 abr. 2010, seção 1, p. 75. 
BRASIL. Saúde repassa R\$ 6,7 milhões para Programa de Plantas Medicinais e Fitoterápicos, Portal Governo do Brasil, jun. 2012. Disponível em: http://www.brasil.gov.br/saude/2012/07/saude-repassa-r6-7-milhoes-para-programa-de-plantas-medicinais-e-fitoterapicos. Acesso em: 22 jun. 2017.

BRASILEIRO, B. G.; PIZZIOLO, V. R.; MATOS, D. S.; GERMANO, A. M.; JAMAL, C. M. Plantas medicinais utilizadas pela população atendida no "Programa de Saúde da Família". Revista brasileira de Ciências Farmacêuticas, Governador Valadares, v. 44, n. 4, p. 629-36, 2008.

CARNIELLO, M. A.; SILVA, R. S.; CRUZ, M. A. B.; GUARIM NETO, G. Quintais urbanos de Mirassol D'OesteMT, Brasil: uma abordagem etnobotânica. Acta Amazonica, Manaus, v. 40, n. 3, p. 451-70, 2010.

CARVALHO, C. A.; MOLINARI, R. F.; SILVA, S. R. S.; PINTO, R.; FANI, M. O. Medicinal plants used by the population of Viçosa, MG. Brasil- preliminary study. Revista Eletrônica de Farmácia, Goiânia, v. 8, n. 4, p. 13-26, 2011.

CASTELLUCCI, S.; LIMA, M. I. S.; NORDI, N.; MARQUES, J. G. W. Plantas medicinais relatadas pela comunidade residente na estação ecológica de Jataí, município de Luís Antônio/SP: uma abordagem etnobotânica. Revista Brasileira de Plantas Medicinais, Paulínia, v. 3, n. 1, p. 51-60, 2000.

COSTA, V. P.; MAYWORM, M. A. S. Plantas medicinais utilizadas pela comunidade do bairro dos Tenentes - município de Extrema, MG, Brasil. Revista Brasileira de Plantas Medicinais, Paulínia, v. 13, n. 3, p. 28292, 2011.

CUNHA, S. A.; BORTOLOTTO, I. M. Etnobotânica de Plantas Medicinais no Assentamento Monjolinho, município de Anastácio, Mato Grosso do Sul, Brasil. Acta Botanica Brasilica, Belo Horizonte, v. 25, n. 3, p. 685-98, 2011.

DI STASI, L. C.; OlIVEIRA, G. P.; CARVALHAES, M. A.; QUEIROZ-JUNIOR, M; TIEN, O. S.; KAKINAMI, S. H., REIS, M. S. Medicinal plants popularly used in the Brazilian Tropical Atlantic Forest. Fitoterapia, v. 73, p. 69-91, 2002.

DORIGONI, P. A.; GHEDINI, P. C.; FRÓES, L. F.; BAPTISTA, K. C.; ETHUR, A. B. M.; BALDISSEROTTO, B.; BURGER, M. E.; ALMEIDA, C. E.; LOPES, A. M. V.; ZÁCHIA, R. A. Levantamento de dados sobre plantas medicinais de uso popular no município de São João do Polêsine, RS, Brasil. I - Relação entre enfermidades e espécies utilizadas. Revista Brasileira de Plantas Medicinais, Paulínia, v. 4, n. 1, p. 69-79, 2001.

FERREIRA, V. L; GODOY, A. G. Importância do estudo e utilização de plantas medicinais, no centro de vivência agroecológico morro das pedras, Belo Horizonte, MG. Revista "Pós em Revista", Belo Horizonte, v. 1, n. 12, p. 103-11, 2016.

FIRMO, W. C. A.; MENEZES, V. J. M.; PASSOS, C. E. C.; DIAS, C. N.; ALVES, L. P. L.; DIAS, I. C. L.; NETO, M. S.; OLEA, R. S. G. Contexto histórico, uso popular e concepção científica sobre plantas medicinais. Caderno de Pesquisa, São Luís, v. 18, n. especial, 2011.

FISCHMAN, L. A.; SKORUPA, L. A.; SOUCCAR, C.; LAPA, A. J. The water extract of coleus barbatus benth decreases gratric secretion in rats. Memórias do Instituto Oswaldo Cruz, Rio de Janeiro, v. 86, p. 141-43, 1991. FREITAS, A. V. L.; COELHO, M. F. B.; MAIA, S. S. S.; AZEVEDO, R. A. B. Plantas medicinais: um estudo etnobotânico nos quintais do Sítio Cruz, São Miguel, Rio Grande do Norte, Brasil. Revista Brasileira de Biociências, Porto Alegre, v. 10, n. 1, p. 48-59, 2012.

FREITAS, A. V. L; COELHO, M. F. B; PEREIRA, Y. B; FREITAS NETO, E. C; AZEVEDO, R. A. B. Diversidade e usos de plantas medicinais nos quintais da comunidade de São João da Várzea em Mossoró, RN. Revista Brasileira de Plantas Medicinais, Paulínia, v. 17, n. 4, p. 845-56, 2015. 
FREITAS, G. G.; COSTA, R. L. G. M.; VENTURIM, N.; COSTA, K. L. Etnosilvicultura de quintais agroflorestais da comunidade quilombola de Abacatal-PA. Embrapa Informação Tecnológica, Brasília, 2010. Disponível em: http://www.sct.embrapa.br/cdagro/tema01/01tema05.pdf. Acesso em: 11 maio 2018.

FUCK, S. B.; ATHANÁZIO, J. C.; LIMA, C. B.; MING, L. C. Plantas medicinais utilizadas na medicina popular por moradores da área urbana de Bandeirantes, PR, Brasil. Semina: Ciências Agrárias, Londrina, v. 26, n. 3, p. 291-96, 2005.

GIRALDI, M.; HANAZAKI, N. Uso e conhecimento tradicional de plantas medicinais no Sertão do Ribeirão, Florianópolis, SC, Brasil. Acta Botânica Brasilica, Belo Horizonte, v. 24, n. 2, p. 395-406, 2010.

HAMILTON, A. Medicinal plant and conservation: issues and approaches. Surrey: International plants Conservation Unit, 2003.

HANAZKI, N.; TAMASHIRO, J. Y.; LEITÃO-FILHO, H. F.; BEGOSSI, A. Diversity of plant uses in two Caiçara communities from the Atlantica Forest coast, Brazil. Biodiversity and Conservation, v. 9, p. 597-615, 2000.

INSTITUTO BRASILEIRO DE GEOGRAFIA E ESTATÍSTICA (IBGE). Pitangui. Brasília, 2017. Disponível em: https:// cidades.ibge.gov.br/brasil/mg/pitangui/panorama. Acesso em: 22 jun. 2017.

KOPPEN, W.; GEIGER, R. Klimate der Erde. Gotha: Verlag Justus Perthes. 1928.

LIMA, R. X.; SILVA, S. M.; KUNIYOSHI, Y. S.; SILVA, L. B. Etnobiolgia de comunidades continentais da área de proteção ambiental de Guaraqueçaba - Paraná - Brasil. Etnológica, Curitiba, v. 4, n. 6, p. 33-55, 2000.

LUCENA, D. S.; LOPES, I. S.; SOUZA, P. F.; TORRES, C. R. M.; MARINHO, M. G. V. Plantas medicinais utilizadas na comunidade urbana de Lagoa, sertão Paraibano. Biofar: Revista de Biologia e Farmácia, v. 9, n. 1, p. 135-45, 2013.

MACEDO, A. F.; OSHIIWA, M.; GUARIDO, C. F. Ocorrência do uso de plantas medicinais por moradores de um bairro do município de Marília - SP. Revista de Ciências Farmacêuticas Básica e Aplicada. Marília, v. 28, n. 1, p. 123-128, 2007.

MARTINS, X. Contribuição para o estudo do valor socioeconómico e cultural das plantas medicinais de Timor-Leste. In: CONGRESSO DA APDEA, ESADR, 8., 2016, Coimbra. Resumos [...]. Coimbra: Universidade de Évora, 2016.

MEIRELLES, L. R.; MEIRELLES, A. L. C. B.; MOTTER, C.; BELLÉ, N. Revista dos Sistemas Agroflorestais. PDA/ PPG7/MMA. Dom Pedro Alcântara: Centro Ecológico. Brasília, 60p. 2003.

MOREIRA, R. C. T.; COSTA, L. C. B.; COSTA, R. C. S.; ROCHA, E. A. Abordagem Etnobotânica acerca do uso de plantas medicinais na Vila Cachoeira, Ilhéus, Bahia, Brasil. Revista Acta Farmacéutica Bonaerense, Argentina, v. 21, n. 3, p. 205-11, 2002.

MOURA, C. L.; ANDRADE, L. H. C. Etnobotânica em quintais urbanos nordestinos: um estudo no bairro da Muribeca, Jaboatão dos Guararapes-PE. Revista Brasileira de Biociências, Porto Alegre, v. 5, n. 1, p. 219-21, 2007.

NASCIMENTO, I. G.; VIEIRA, M. R. S. Manual de Plantas Medicinais - Farmácia Verde. Católica Unisantos, 2014. Disponível em: www.unisantos.br/wp-content/uploads/2014/02/farmacia-verde-livro.pdf. Acesso em: 7 ago. 2017. 
NOBRE, C. J. S. Etnobotânica de plantas medicinais no ensino de Biologia: uma contribuição da escola pública rumo ao resgate do conhecimento popular. 2015. 79p. Monografia (Graduação em Biologia) Universidade Federal de Campina Grande, Patos, 2015.

OLIVEIRA, E. R.; MENINI NETO, L. Levantamento etnobotânico de plantas medicinais utilizadas pelos moradores do povoado de Manejo, Lima Duarte-MG. Revista Brasileira de Plantas Medicinais, Paulínia, v. 14, n. 2, p. 311-20, 2012.

OLIVEIRA, H. B.; KFFURI, C. W.; CASALI, V. W. D. Ethnopharmacological study of medicinal plants used in Rosário da Limeira, Minas Gerais, Brazil. Revista Brasileira de Farmacognosia, Curitiba, v. 20, n. 2, p. 256-60, 2010.

OLIVEIRA, O. F. V.; GONDIM, M. J. C. Plantas medicinais utilizadas pela população de Caldas Novas, GO e o conhecimento popular sobre a faveira (Dimorphandra mollis Benth-Mimosoideae). Revista Brasileira de Agroecologia, Porto Alegre, v. 8, n. 1, p. 156-69, 2013.

PILLA, M. A. C.; AMOROZO, M. C. M.; FURLAN, A. Obtenção e uso de plantas medicinais no distrito de Martim Francisco, Município de Mogi-Mirim, SP, Brasil. Acta Botanica Brasilica, Belo Horizonte, v. 20, n. 4, p. 789-802, 2006.

RODRIGUES, A. G.; CASALI, V. W. D. Plantas medicinais, conhecimento popular e etnociência. 2002. In: RODRIGUES, A. G.; ANDRADE, F. M. C.; COELHO, F. M. G. Plantas medicinais e aromáticas: etnoecologia e etnofarmacologia. Viçosa: Universidade Federal de Viçosa, 2002. p. 25-76.

RODRIGUES, A. C. C.; GUEDES, M. L. S. Utilização de plantas medicinais no Povoado Sapucaia, Cruz das Almas-Bahia. Revista Brasileira de Plantas Medicinais, Paulínia, v. 8, n. 2, p. 1-7, 2006.

SANTOS, A. B. N.; ARAÚJO, M. P.; SOUSA, R. S.; LEMOS, J. R. Plantas medicinais conhecidas na zona urbana de Cajueiro da Praia, Piauí, Nordeste do Brasil. Revista Brasileira de Plantas Medicinais, Paulínia, v. 18, n. 2, p. 442-50, 2016.

SANTOS, E. D.; FARIA, M. T; VILHALVA, D. A. A. Levantamento etnobotânico das plantas medicinais utilizadas pela população do residencial Goiânia Viva, Região Noroeste - Goiânia, Goiás, Brasil. Revista Eletrônica de Educação da Faculdade Araguaia, v. 7, p. 13-40, 2015.

SILVA, C. G.; MARINHO, M. G. V.; LUCENA, M. F. A.; COSTA, J. G. M. Levantamento etnobotânico de plantas medicinais em área de Caatinga na comunidade do Sítio de Nazaré, município de Milagres, Ceará, Brasil. Revista Brasileira de Plantas Medicinais, Paulínia, v. 17, n. 1, p. 133-42, 2015.

SILVA, R. M.; FARIA, M. T. Caracterização etnobotânica e histoquímica de plantas medicinais utilizadas pelos moradores do bairro Carrilho, Goianésia (GO). Enciclopédia Biosfera, Goiânia, v. 10, n. 19, p. 2807-29, 2014.

SILVA, R. C.o; SANTAN, A. D; SANTOS, A. A. P; CERQUEIRA, G. S. Plantas medicinais utilizadas na saúde da mulher: riscos na gravidez. Diálogos \& Ciência, Slavador, v. 32, p. 243-46, 2012.

SIVIERO, A.; DELUNARDO, T. A.; HAVERROTH, M.; OLIVEIRA L. C.; MENDONÇA, A. M. S. Plantas medicinais em quintais urbanos de Rio Branco, Acre. Revista Brasileira de Plantas Medicinais, Paulínia, v. 14, n. 4, p. 598-610, 2012.

VEIGA JUNIOR, V. F. Estudo do consumo de plantas medicinais na Região Centro-Norte do Estado do Rio de Janeiro: aceitação pelos profissionais de saúde e modo de uso pela população. Revista Brasileira de Farmacognosia, Curitiba, v. 18, n. 2, p. 308-13, 2008. 


\section{Sobre os autores:}

Filipe Rodrigues Valeriano - Mestre em Produção Vegetal pela Universidade Federal dos Vales do Jequitinhonha e Mucuri (UFVJM). E-mail: filiper.valeriano@hotmail.com, Orcid: http://orcid.org/0000-0003-1656-759X

Fabiana Ramos Savani - Mestre em Ciência e Tecnologia Ambiental pela Universidade Federal do ABC (UFABC). E-mail: fabianasavani@gmail.com, Orcid: http://orcid.org/0000-0002-5267-2779 Maria Rejane Valeriano da Silva - Graduada em História pela Faculdade de Pará de Minas (FAPAM). E-mail: varci@yahoo.com.br, Orcid: http://orcid.org/0000-0001-6554-586X 
